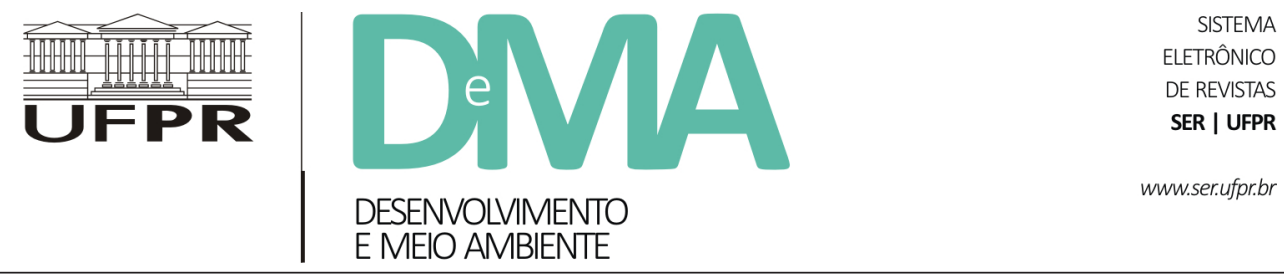

\title{
Informação de base ecossistêmica como ferramenta de apoio à gestão costeira integrada da Ilha de Santa Catarina, Brasil
}

\author{
Ecosystem-Based Information as a Support Tool to the Integrated \\ Coastal Management of the Santa Catarina Island, Brazil
}

Andre de Souza de LIMA ${ }^{1 *}$, Apoena Calixto FIGUEIROA ${ }^{1}$, Tiago Borges Ribeiro GANDRA², Bruno Henrique Moreira PEREZ ${ }^{1}$, Bruno Andrade Queiroz dos SANTOS ${ }^{1}$, Marinez Eymael Garcia SCHERER ${ }^{1}$

${ }^{1}$ Universidade Federal de Santa Catarina (UFSC), Florianópolis, SC, Brasil.

${ }^{2}$ Instituto Federal de Educação, Ciência e Tecnologia do Rio Grande do Sul (IFRS), Rio Grande, RS, Brasil.

*E-mail de contato: geoandrelima@gmail.com

Artigo recebido em 30 de agosto de 2017, versão final aceita em 14 de novembro de 2017.

RESUMO: Os ecossistemas contribuem, por meio de seus Serviços Ecossistêmicos (SEs) diretos ou indiretos, para o bemestar humano. Desse modo, para que os ecossistemas mantenham uma condição saudável, a gestão com base em ecossistemas aborda as interações homem-meio, para que seus serviços sejam apropriados de maneira sustentável. O presente trabalho tem como objetivo dar subsídios para uma gestão costeira integrada da Ilha de Santa Catarina (ISC) e entorno, município de Florianópolis, com base em uma perspectiva ecossistêmica. Para isso, foram necessários o mapeamento de ecossistemas, a elaboração e a análise de uma matriz de serviços, e a definição dos serviços básicos para o bem-estar humano, ou seja, os serviços que possuem maior capacidade de contribuir para um meio de vida seguro e adequado. $\mathrm{O}$ mapeamento realizado possibilitou a classificação de 14 ecossistemas na área de estudo. Os ecossistemas marinhos correspondem a 51,9\% e os terrestres a 37,3\%. As áreas antropizadas urbanas e rurais correspondem a 10,8\% da área de estudo. Foram identificados seis SE básicos na ISC, associados a 11 ecossistemas mapeados: estoque pesqueiro, proteção da linha de costa, recarga de aquífero, recurso hídrico, depuração de poluentes e berçário natural. O Sistema de Informações criado permitiu a realização de consultas quantitativas e qualitativas sobre os ecossistemas e seus serviços e o aperfeiçoamento dos dados apresentados na matriz de sistemas e serviços já existente. Além disso, estabeleceu relações espaciais entre sistemas e serviços, dando condição a futuros trabalhos de pesquisa na região.

Palavras-chave: serviços ecossistêmicos; gestão com base ecossistêmica; ecossistemas. 
ABSTRACT: Ecosystems contribute, through direct or indirect Ecosystem Services (ES), for the human well-being. Thus, to maintain ecosystems healthy condition, ecosystem-based management addresses interactions between human activities and environment, focusing on the sustainable use of these services. The aim of this research is to provide support for an integrated coastal management, based on an ecosystemic perspective, for Ilha de Santa Catarina (ISC) and surrounding bays and sea areas, Florianópolis municipality. To reach this goal it is necessary to map the island ecosystems, to analyze the ecosystem services, and to define those services that are basic for the human well-being, that is, services that have the greatest capacity to contribute to a safe and adequate livelihood. The mapping allowed the classification of 14 ecosystems. Marine ecosystems correspond to $51.9 \%$ of the study area and the terrestrial ones to $37.3 \%$. The anthropogenic urban and rural areas correspond to $10.8 \%$ of the study area. Six basic ESs were identified in the ISC: fish stocks, shoreline protection, aquifer recharge, fresh water supply, pollution mitigation and natural nursery; those associated to 11 mapped ecosystems. The Information System created allowed quantitative and qualitative exploration on ecosystems and their services and improved already existing data. In addition, it established spatial relationships between systems and services, allowing conditions to future research in the region.

Keywords: ecosystem services; ecosystem based management; ecosystems.

\section{Introdução}

A costa brasileira possui $8.698 \mathrm{~km}$ de extensão, representando $32 \%$ da fronteiras nacionais (Scherer et al., 2010). Além disso, os 395 municípios que compõem a zona costeira brasileira concentram cerca de 45 milhões de pessoas, pelos quais circula metade do PIB nacional (MMA, 2015). Essas características fazem com que os ecossistemas inseridos nessas áreas sofram forte pressão das intensas atividades antrópicas (Gruber et al., 2011), sobretudo os ecossistemas adjacentes às áreas urbanas, os quais até 2050 concentrarão mais de dois terços da população mundial (UN, 2010).

Florianópolis, capital do Estado de Santa Catarina, é um exemplo de município costeiro no qual os ecossistemas vêm sofrendo estas pressões (Diederichsen et al., 2013; Oliveira et al., 2016). Seu território compreende uma pequena porção continental e a Ilha de Santa Catarina, a qual possui sua vertente oeste abrigada e as vertentes norte e leste expostas à ação de ondas (Klein et al., 2016).
Para que os ecossistemas mantenham uma condição saudável, a Gestão com Base em Ecossistemas (GBE) aborda as interações homem-meio de maneira que os serviços providos por estes ecossistemas (Serviços Ecossistêmicos-SEs) sejam apropriados de modo sustentável (Mcleod et al., 2005). A GBE também explora o conceito de trade-offs (Granek et al., 2010), analisando e propondo ações referentes a alterações de ambientes e troca de SE naturais por aqueles antrópicos.

De acordo com Odum (1956), um ecossistema é caracterizado pela totalidade de uma área determinada na qual interagem seres vivos e não vivos, ou seja, inclui uma diversidade biótica, bem como o ciclo de materiais e a estrutura trófica. É importante ressaltar que cada uma das variáveis condiciona a existência da outra em um ecossistema. Já Chistofoletti (1999) afirma que um ecossistema pode ser classificado como uma área homogênea de interação de organismos físicos com seu ambiente.

Para implementar a GBE e avaliar a complexidade que envolve os ecossistemas, é necessário 
compreender as funções e os processos que condicionam os bens e serviços para o bem-estar humano (Figura 1). Esses processos estão relacionados às interações complexas envolvendo forças motrizes de matéria e energia, dando condição às funções que refletem na capacidade dos ecossistemas em gerar seus bens e serviços, tais como a função de habitat para refúgio e reprodução de espécies; função de produção de fontes energéticas por meio da transformação; função de informação, onde se enquadram o desenvolvimento cognitivo, a reflexão e as religiões e crenças (De Groot et al., 2002).

Portanto, os ecossistemas contribuem, por meio de seus Serviços Ecossistêmicos (SEs) diretos ou indiretos, para o bem-estar humano (Costanza et al., 1997; De Groot et al., 2010; Braat \& De Groot, 2012; Böhnke-Henrichs et al., 2013).
No contexto de identificação das estruturas e dos processos relacionados aos serviços ecossistêmicos, o mapeamento dos ecossistemas que estruturam estes processos é etapa fundamental para a GBE (Scherer \& Asmus, 2016).

Um campo importante de aplicação da GBE é a Gestão Costeira Integrada (GCI), definida como um processo consciente de gestão dos usos do litoral e do oceano em relação aos seus respectivos sistemas biofísicos (Cicin-Sain \& Knecht, 1998). A GCI é a base do Plano Nacional de Gerenciamento Costeiro - PNGC (Brasil, 1988; CIRM, 1997; Brasil, 2004), o qual não exige necessariamente uma base ecossistêmica na construção de suas diretrizes.

Muitas vezes a ocupação da zona costeira é pautada por aspectos demográficos associados ao desenvolvimento econômico, sem considerar o que

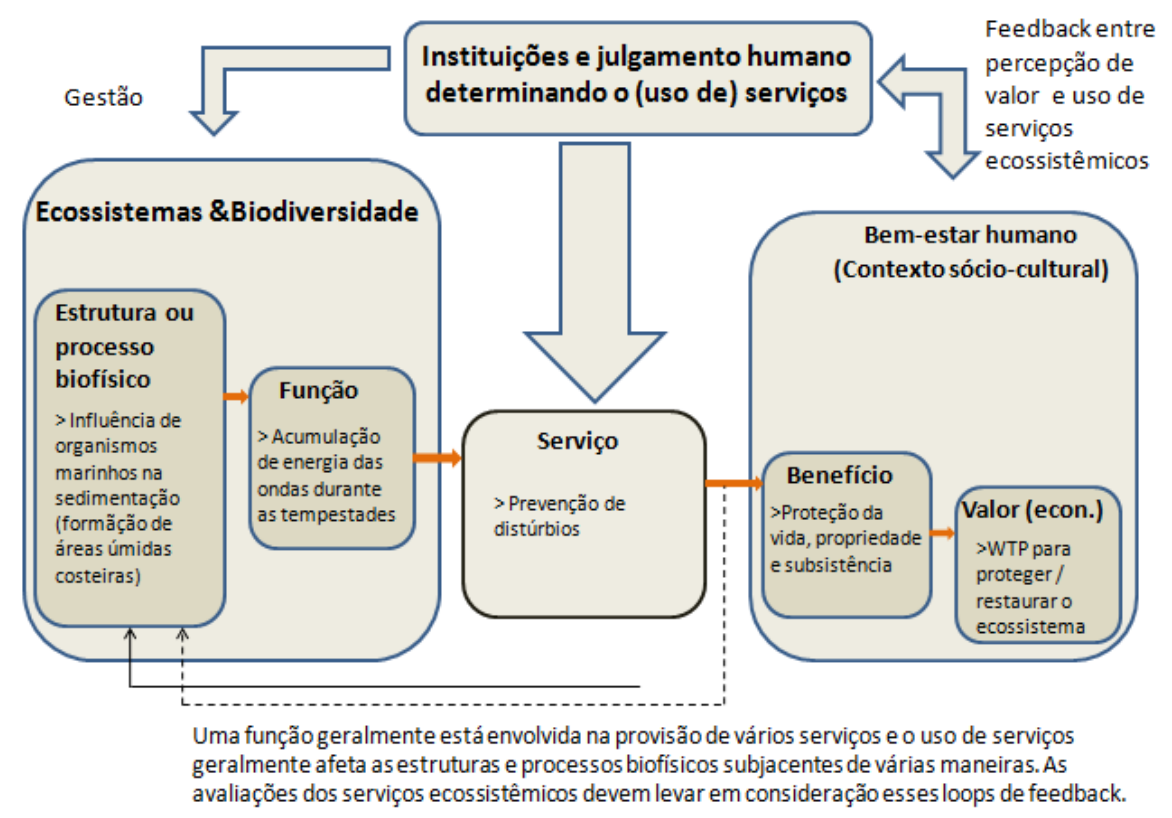

FIGURA 1 - Relação entre a abordagem dos SEs e o bem-estar humano.

FONTE: Adaptado de Böhnke-Henrichs et al. (2013).

Desenvolv. Meio Ambiente, v. 44, Edição especial: X Encontro Nacional de Gerenciamento Costeiro, p. 20-35, fevereiro 2018. 
preconizam os instrumentos do PNGC no Brasil, como o Zoneamento Ecológico-Econômico (Asmus \& Kitzmann, 2004; Asmus et al., 2006; Barragán, 2014). Ou seja, a atual gestão territorial aplicada aos municípios costeiros parte do pressuposto que um determinado espaço deve abrigar um número previsto de habitantes e determinadas atividades que são de interesse para a sociedade ou parte dela, mesmo que em detrimento de seus ecossistemas e serviços (Figueiroa \& Scherer, 2016).
Nesse modelo, os ecossistemas devem se adaptar a essas condições preestabelecidas, ignorando por vezes as consequências negativas futuras, as quais são tratadas somente quando se manifestam, a depender da tecnologia então existente, normalmente com grandes custos energéticos e ecossistêmicos. Esses custos, por sua vez, geram novas consequências negativas, perpetuando o ciclo que atualmente predomina em nossa sociedade (Neal et al., 2017).
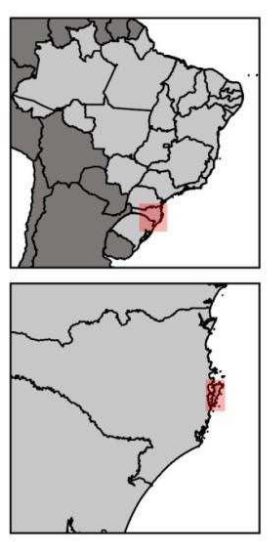

Legenda

Área de Estudo
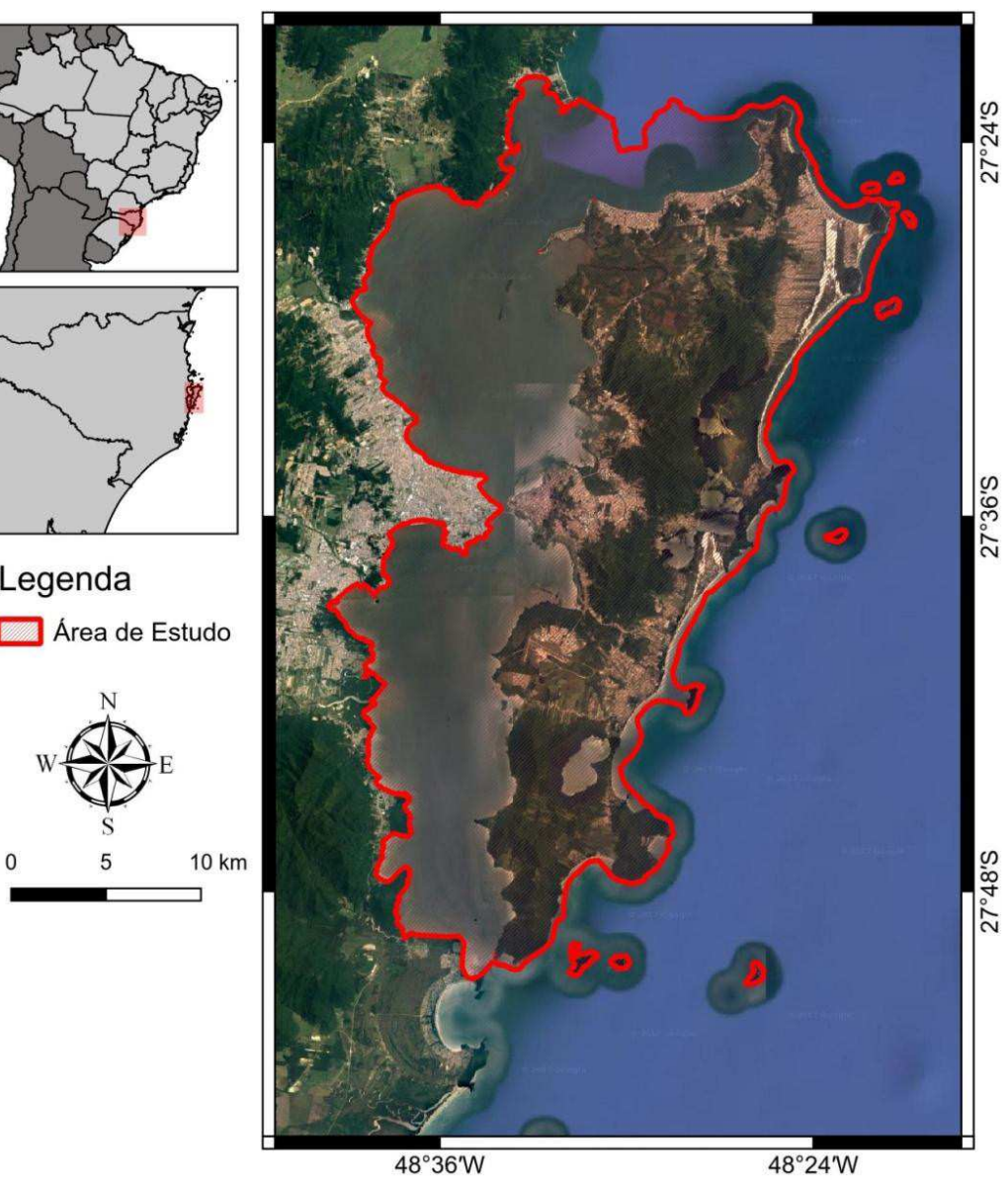

FIGURA 2 - Localização da área de estudo. 
Ou seja, ao não focar sua implantação nas características ecossistêmicas, o atual modelo de gestão da zona costeira deixa de trazer consigo importantes limites de uso e ocupação, que se traduzem ao longo do tempo em degradação de ecossistemas e da própria sociedade pela ausência ou insuficiência de serviços ecossistêmicos outrora existentes (Asmus et al., 2006; Barragán, 2014; Neal et al., 2017).

Assim, este trabalho mapeou os ecossistemas da Ilha de Santa Catarina (ISC), assim como os do setor marinho adjacente e das baías Norte e Sul no município de Florianópolis (Figura 2). Tratou também de relacioná-los aos SEs advindos desses ambientes, sobretudo os básicos para o bem-estar humano. Teve assim como objetivo dar subsídios para uma gestão costeira integrada da ISC, Florianópolis, com base em uma perspectiva ecossistêmica.

\section{Material e métodos}

Foram necessárias três etapas para a elaboração do presente artigo: a) mapeamento dos ecossistemas da Ilha de Santa Catarina e entorno; b) elaboração da matriz de SE; e c) definição dos SE básicos para o bem-estar humano.

Para contextualizar a área de estudo, foi realizado o mapeamento dos ecossistemas conforme propõem Erhard et al. (2017) baseando-se na análise dos SEs da Ilha de Santa Catarina apresentada por Scherer \& Asmus (2016). A determinação dos SEs básicos para o bem-estar humano na área de estudo foi realizada de acordo com MEA (2005).

O mapeamento dos ecossistemas da Ilha de Santa Catarina foi iniciado em ambiente SIG, utilizando o software QGIS 2.18.9 por meio de fotointerpretação e vetorização dos ecossistemas (SDS, 2010; Erhard et al., 2017). O refinamento do mapa produzido nesta primeira fase foi realizado com apoio em bases cartográficas secundárias e com informações altimétricas obtidas em Modelo Digital de Terreno (SDS, 2010), formações geológicas (Tomazzoli \& Pellerin, 2015) e cobertura vegetal, cedido pela Gerência de Cartografia do Instituto de Planejamento Urbano de Florianópolis (dados não publicados). Foram também realizados levantamentos de campo para consolidação das informações mapeadas.

A geometria básica dos ecossistemas foi obtida em escala fixa de 1:25.000. No entanto, o refinamento da base cartográfica foi apoiado em mapeamentos de diversas escalas e resoluções espaciais, sobretudo de maior precisão.

O diagnóstico dos SEs da ISC teve como base a matriz conceitual apresentada por Scherer \& Asmus (2016) que, por meio de uma abordagem ambiental sistêmica, consideraram os seguintes aspectos: (1) sistemas ambientais/ecossistemas, (2) tipos de serviços (De Groot et al., 2002), (3) os principais serviços ecossistêmicos, (4) os principais benefícios, (5) os atores sociais beneficiados, e (6) as principais pressões ambientais.

Embora Scherer \& Asmus (2016) tenham utilizado Sistemas Ambientais (Christofoletti, 1999) como unidade de análise, integrando as áreas antropizadas e ocupadas em sua pesquisa, o presente artigo foca nos ecossistemas, ou seja, nos ambientes naturais, objeto da GBE.

No que tange aos SE, ressalta-se que habitat e ciclagem de nutrientes foram categorizados como funções dos ecossistemas, pois não representam benefícios diretos ou indiretos ao bem-estar humano (Costanza et al., 1997; De Groot et al., 2002), 
ainda que alguns autores considerem estas funções ecossistêmicas como serviços de "Suporte" (MEA, 2005; Scherer \& Asmus, 2016). Assim, a presente pesquisa considerou SEs aqueles que contribuem direta ou indiretamente para o bem-estar humano.

Foram também consideradas análises pontuais envolvendo ecossistemas e serviços na Ilha de Santa Catarina (Figueiroa et al., 2016; Figueiroa \& Scherer, 2016; Veiga Lima et al., 2016), que ressaltam a relação direta entre ecossistemas e o bem-estar humano da população de Florianópolis, assim como a necessidade de integrar a base ecossistêmica ao atual modelo de gestão costeira.

A matriz de serviços ecossistêmicos apresentada em Scherer \& Asmus (2016) e que tem como base o trabalho do Laboratório de Gestão Costeira Integrada (LAGECI) da Universidade de Santa Catarina (UFSC), assim como o mapa dos ecossistemas da ISC, elaborado na presente pesquisa, foram importados para um Sistema Gerenciador de Banco de Dados (SGBD) PostgreSQL 9.3, instalado em um servidor com acesso externo, que permite a utilização do banco de dados por diversos usuários em qualquer computador com acesso à internet. $\mathrm{O}$ SGBD permite a construção de relacionamentos entre as diversas tabelas e as geometrias espaciais, bem como a realização de consultas e análise dos dados. Os mapas foram elaborados no software QGIS 2.18.9 e as análises estatísticas no R. Os dois softwares podem acessar de forma remota o SGBD.

Foi realizada uma análise de cluster com o objetivo de agrupar os ecossistemas semelhantes em relação aos SEs associados. Os SEs de cada ecossistema foram transformados em valores binários ( 0 - ausência e 1 - presença) para a análise de cluster utilizando o índice Jaccard e o cálculo de distância para variáveis categóricas binárias.
Os SEs que apresentam maior intensidade em relação com o bem-estar humano, assim como em relação ao maior potencial de mediação por fatores econômicos (Figura 3) conforme MEA (2005), foram denominados "Serviços Ecossistêmicos Básicos". Assim como abordado por Scherer \& Asmus (2016), para identificação desses SEs, foi utilizada opinião especialista (Oliveira \& Corona, 2008; Krueger et al., 2012; Martin et al., 2012; Ban et al., 2015) dos membros do Laboratório de Gestão Costeira Integrada (LAGECI) da Universidade Federal de Santa Catarina (UFSC).

Devido ao grande número de SEs identificados para a área de estudo, a classificação dos SEs básicos buscou direcionar atenção aos serviços que possuem maior capacidade de contribuir para um meio de vida seguro e adequado, tais como comida e água suficientes em todos os momentos. $\mathrm{O}$ acesso a esses recursos é fortemente mediado por circunstâncias socioeconômicas (MEA, 2005) e que, portanto, sofrem maior pressão antrópica.

\section{Resultados e discussão}

O mapeamento realizado possibilitou a classificação de 14 ecossistemas na Ilha de Santa Catarina (Figura 4). Os ecossistemas marinhos correspondem a $51,9 \%$ da área de estudo e os terrestres a 37,3\%. As áreas antropizadas urbanas e rurais correspondem juntas a $10,8 \%$ da área de estudo. Com relação à representatividade dos ecossistemas, ou seja, das áreas naturais não antropizadas da área de estudo, é importante salientar que, apesar de representarem $89,2 \%$ da área de estudo, as baías representam $43,4 \%$ e o ecossistema marinho adjacente (delimitado entre a linha de costa e a isóbata de 10m), 14,8\%. 
Desse modo, a extensão dos ecossistemas marinhos, se utilizada individualmente como parâmetro para priorização de ecossistemas, em relação a medidas de planejamento e gestão, pode não expressar a realidade da zona costeira.

É importante salientar que o contexto e o objetivo do mapeamento, que visam a uma perspectiva ecossistêmica em nível municipal, exigiram a generalização de algumas feições do ambiente, a fim de compatibilizar a complexidade dos ecossistemas e o contexto de uso planejado. Cabe ressaltar que a relação intrínseca entre os ecossistemas e seus processos faz com que sejam necessários diversos níveis de escala, inclusive temporais, para compreensão de seu funcionamento (Hein et al., 2006). A escala, por ser um fator limitante durante um mapeamento, faz com que nem todos os detalhes da área de estudo sejam visualizáveis (Traun et al., 2017).

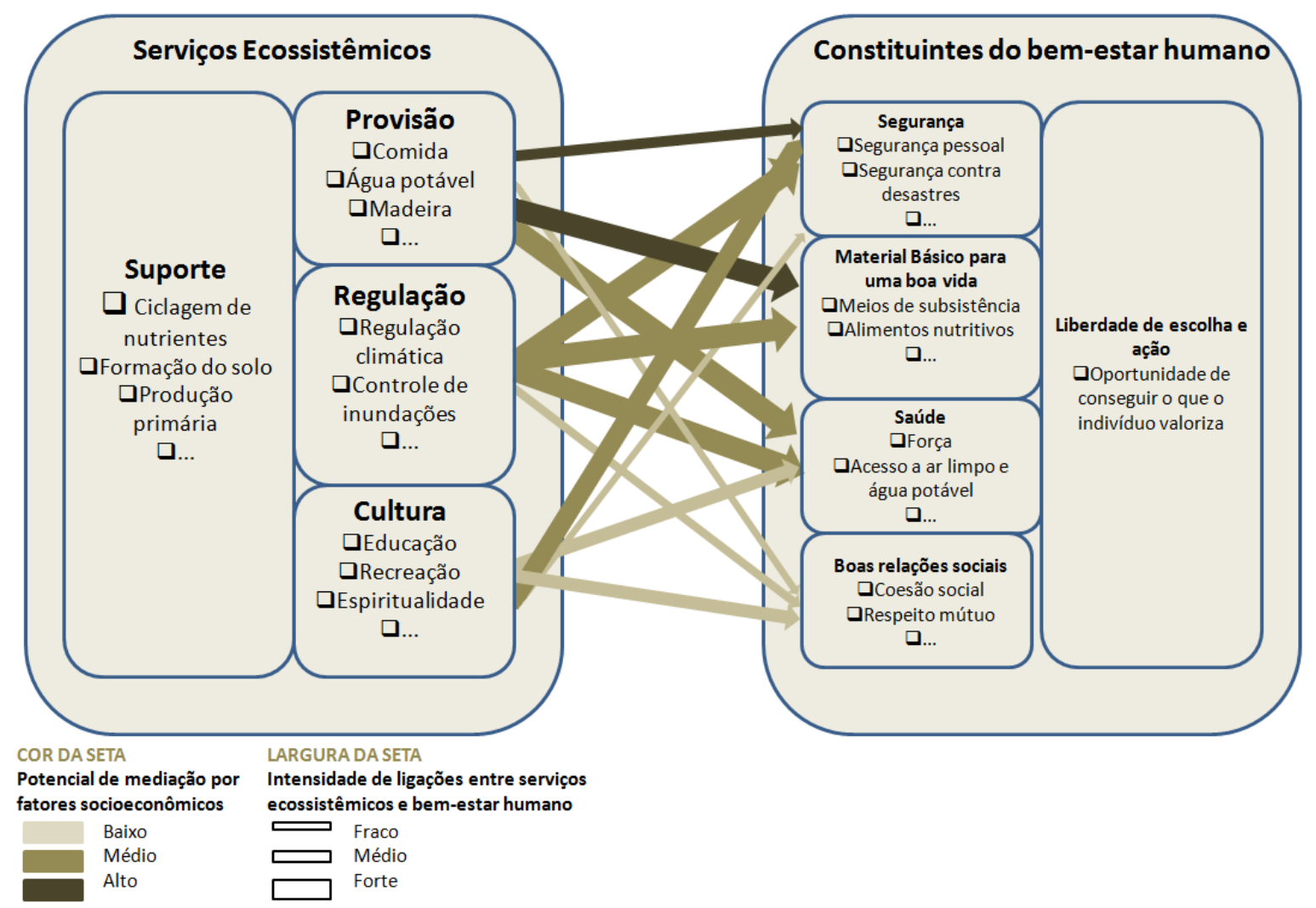

FIGURA 3 - Relação entre os SEs e o bem-estar humano.

FONTE: Adaptado de MEA (2005). 


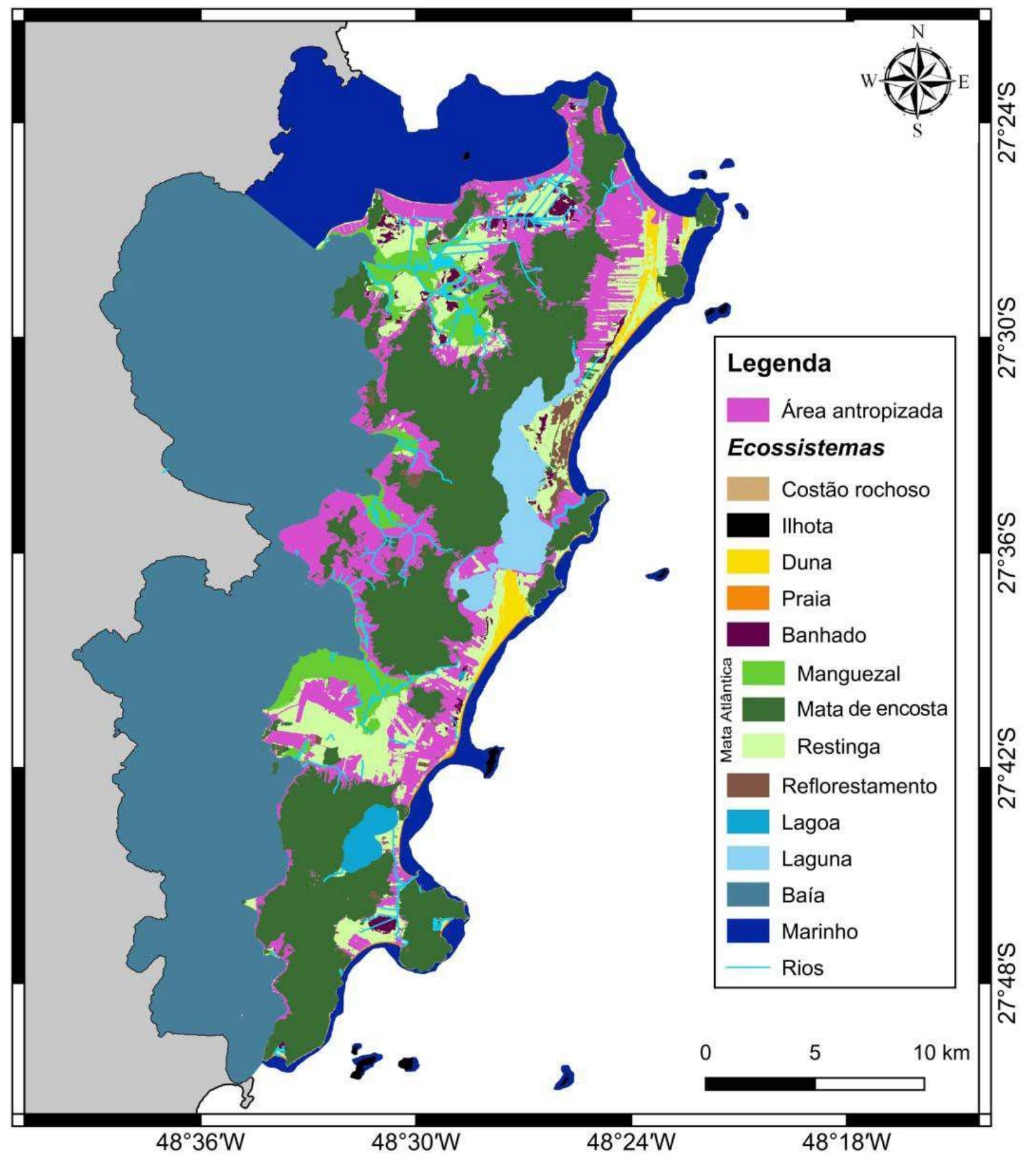

FIGURA 4 - Ecossistemas da Ilha de Santa Catarina e entorno. 
Considerando apenas o ambiente terrestre, os ecossistemas naturais representam atualmente $77,6 \%$ da Ilha de Santa Catarina. A Mata Atlântica (Floresta Ombrófila Densa de encosta) representa a maior extensão (44,5\%), seguida pelas áreas de restinga (14\%), laguna $(4,8 \%)$, manguezais $(6,7 \%)$, banhados $(1,9 \%)$ e dunas $(1,6 \%)$. As lagoas, ilhotas, praias, costões rochosos e áreas de reflorestamento totalizam $4,1 \%$ dos ecossistemas terrestres. Por características intrínsecas, os rios foram mapeados como feições lineares e, portanto, não foram inseridos nos cálculos de áreas, mas foram considerados na descrição dos SEs.
Com relação ao ecossistema terrestre de maior representatividade, a Mata de encosta é um ecossistema sob grande pressão antrópica (Scarano \& Ceotto, 2015). A zona costeira brasileira concentra porção residual equivalente a $8 \%$ de sua extensão original. Todavia, por conta da atual legislação, cerca de metade das áreas remanescentes associadas a Unidades de Conservação (UCs) encontra-se em estágio de regeneração (Colombo \& Joly, 2010).

Com relação às atuais políticas de proteção das áreas naturais costeiras, atualmente todos os ecossistemas terrestres identificados na área de estudo são protegidos de alguma forma pela legislação vi-

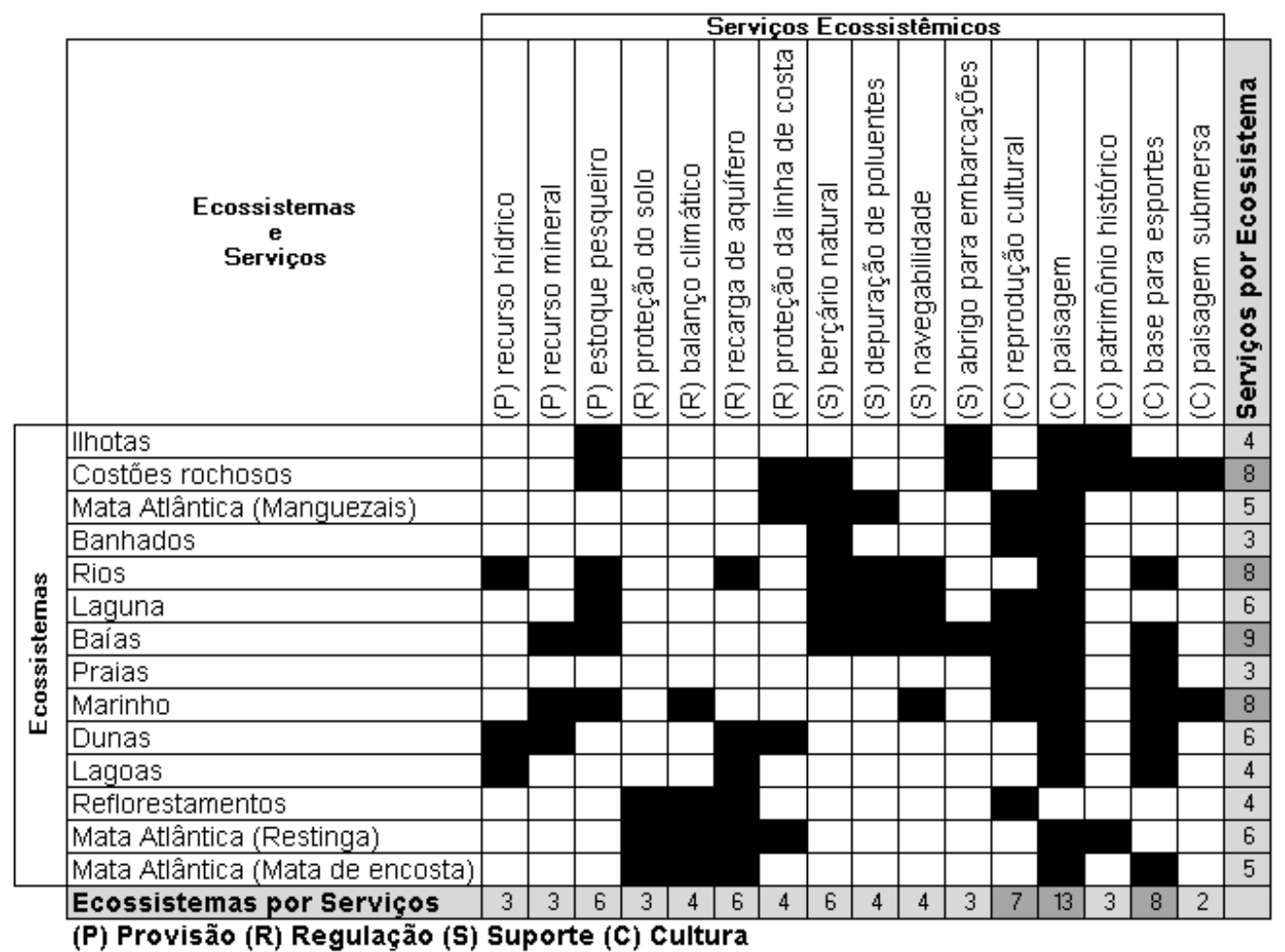

FIGURA 5 - Matriz de distribuição dos SEs em relação aos ecossistemas mapeados. 
gente, mesmo que em diferentes níveis de proteção. Não obstante, existem 18 Unidades de Conservação (UCs) na Ilha de Santa Catarina, distribuídas em $21,2 \%$ dos ecossistemas terrestres e em $8,8 \%$ dos ecossistemas marinhos do da área de estudo, sendo sete UCs municipais, três estaduais e quatro federais; além disso, existem duas reservas particulares (RPPNs) e duas UCs sem normativas legais. As UCs classificam 11 ecossistemas em diversas categorias de áreas protegidas (Ferretti \& Beltrame, 2010; Figueiroa et al., 2016; ICMBio, 2016).

De acordo com Scherer \& Asmus (2016), foram identificados 47 SEs na Ilha de Santa Catarina, os quais são ameaçados por 39 vetores de pressão antrópica. No entanto, considerando apenas as contribuições de áreas não antropizadas e após a reclassificação das funções e SEs, foram considerados 16 SEs para a área de estudo (Figura 5).

Foram considerados três serviços de provisão, quatro de regulação, quatro de suporte e cinco serviços culturais. Conforme representado na Tabela 1 , os SEs culturais estão associados à maior parte dos ecossistemas, entretanto, os serviços básicos para o bem-estar humano têm relação a um número menor de ecossistemas (ex.: recurso hídrico). Ainda, os ecossistemas "Rios", "Baía", "Costão rochoso" e "Marinho" destacam-se por contribuírem com o maior número de SEs em relação aos demais ecossistemas mapeados.

Os ecossistemas responsáveis pelos serviços de provisão, principalmente os relacionados à água potável e aos alimentos, quando alterados, impactam de maneira substancial na manutenção do bem-estar humano. Cabe ressaltar que o acesso a estes bens é fortemente mediado por circunstâncias socioeconômicas. Os SEs de suporte e regulação têm influência em menor escala, todavia, têm forte ligação aos SEs de provisão, portanto, alterações nesses serviços surtem impactos indiretos ao bem-estar humano. Já os SEs culturais tem vínculos relativamente fracos com os elementos principais do bem-estar humano (MEA, 2005).

Foram identificados seis SEs básicos na ISC: estoque pesqueiro (Provisão), proteção da linha de costa (Regulação), recarga de aquífero (Regulação), recurso hídrico (Provisão), depuração de poluentes (Regulação) e berçário natural (Suporte) (Tabela 1). Os SEs básicos estão associados a 11 ecossistemas mapeados, sendo que os rios são responsáveis por quatro SEs básicos; já os costões rochosos, manguezais, dunas e baías estão associados a três SEs básicos. Restinga, mata de encosta, lagoas e lagunas estão associadas a dois serviços básicos.

A análise de similaridade dos ecossistemas baseada em seus serviços resultou em cinco clusters (Figura 6). Além da similaridade, esses clusters identificam os ecossistemas que estão conectados em relação à classe de serviços oferecidos para o bem-estar humano, ou seja, ficando evidentes quais os ecossistemas-chave para os serviços de provisão, regulação e suporte, facilitando o direcionamento para políticas públicas de conservação que considerem de maneira sistêmica a importância dos SEs da ISC.

Essa análise de similaridade permite identificar a relação entre os diferentes ecossistemas, levando à melhor compreensão dos fluxos ambientais entre eles na produção dos SEs. Por exemplo, os ecossistemas aquáticos (exceto lagoas) ficaram agrupados junto com as praias, pois têm a característica de ambientes que dão suporte a outros serviços. Já as dunas e lagoas se agruparam principalmente devido à sua importância como fonte de água potável (provisão) e para a recarga de aquíferos (regulação). 
Ressalta-se que no norte da ISC a água potável é possuem em comum os serviços de proteção do proveniente das dunas e em sua porção sul, da Lagoa solo, balanço climático e recarga de aquífero, todos do Peri. Reflorestamento, restinga e mata atlântica considerados como serviços de regulação.

TABELA 1 - Serviços Ecossistêmicos básicos identificados na área de estudo. (P) Provisão (R) Regulação (S) Suporte (C) Cultura.

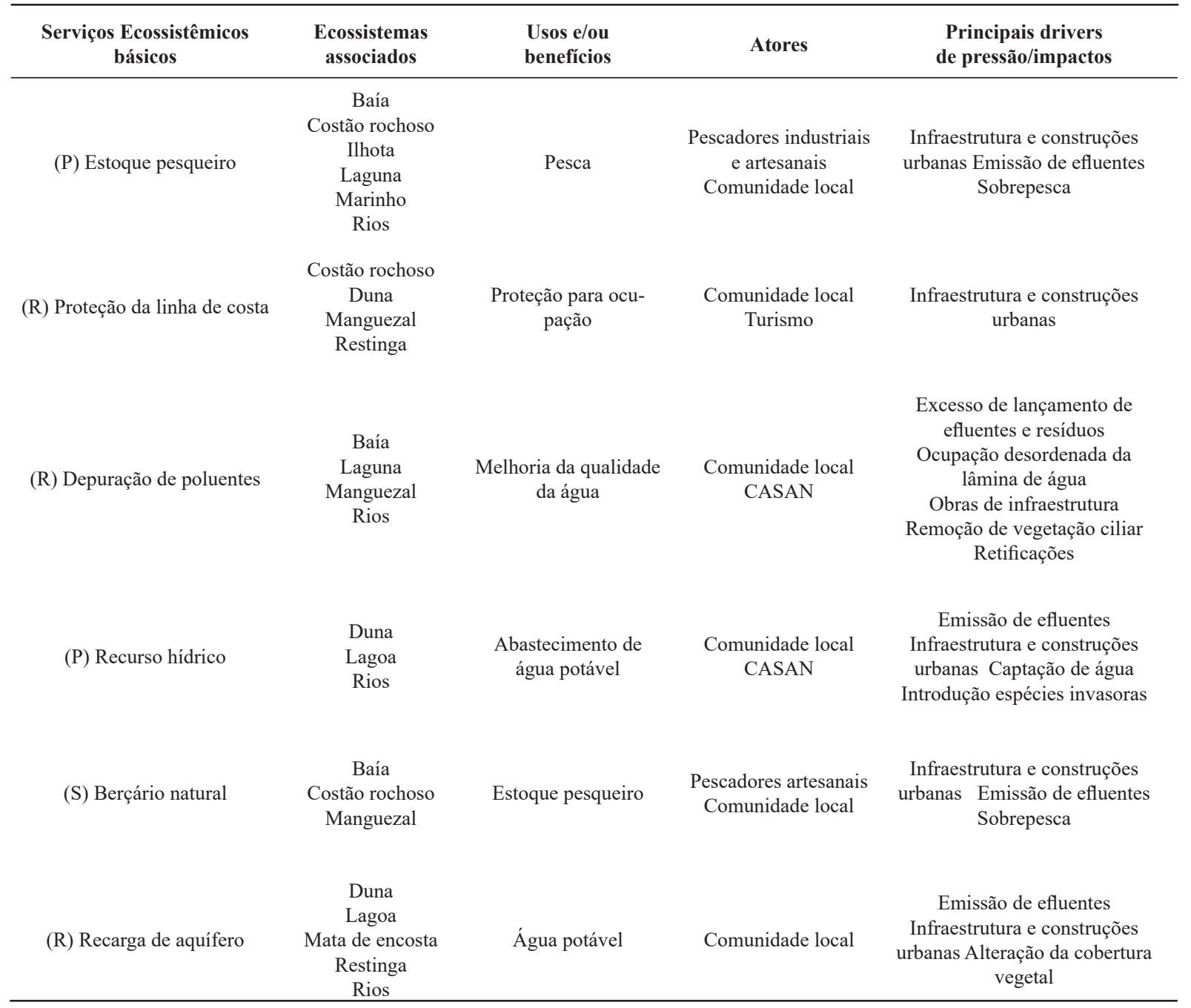




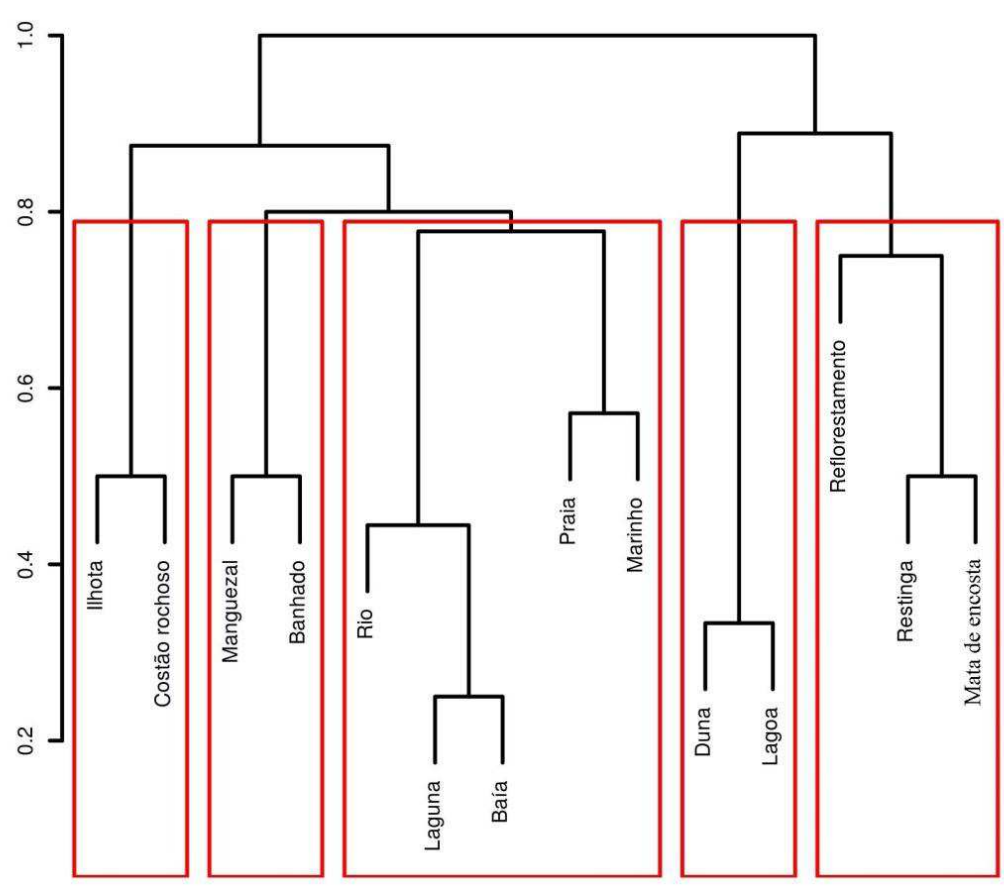

FIGURA 6 - Agrupamento de Ecossistemas por similaridade.

\section{Conclusões}

O Sistema de Informações criado para o mapeamento (Figura 5) permitiu a realização de consultas quantitativas e qualitativas sobre os ecossistemas e seus serviços na Ilha de Santa Catarina e entorno. Também permitiu aperfeiçoar os dados apresentados na tabela de ecossistemas e serviços elaborada anteriormente (Scherer \& Asmus, 2016). Além disso, estabeleceu relações espaciais entre ecossistemas e serviços, dando condição a futuros trabalhos de pesquisa na região.
Uma vez relacionados os ecossistemas e seus serviços, torna-se possível a criação de cenários que tenham como objetivo identificar os SE potencialmente afetados em uma situação de impacto aos ecossistemas existentes na Ilha de Santa Catarina. Ao ligar os serviços, os benefícios e as partes interessadas nos benefícios (atores), a matriz dos Serviços de Ecossistemas pode também ser usada para a identificação das principais partes interessadas que devem ser envolvidas em um processo de planejamento e gerenciamento participativo. Essa ação participativa é indubitavelmente facilitada 
quando os atores sociais contam com uma ferramenta metodológica que demonstra os benefícios do meio ambiente, abordando eventualmente interesses individuais e coletivos (Scherer \& Asmus, 2016).

Relacionar os SEs com os ecossistemas pode também se tornar uma importante ferramenta para analisar o ambiente, considerando de maneira integrada os aspectos ecológicos, econômicos e sociais, uma vez que as contribuições dos ecossistemas por meio de bens e serviços são traduzidas para a gestão por meio de uma interlocução científica. As decisões tomadas pelos gestores, além de regularem um uso sustentável desses serviços, podem priorizar o equilíbrio entre o bem-estar humano e a integridade dos ecossistemas (Scherer \& Asmus, 2016).

Também é importante salientar que essa abordagem envolve justiça social, uma vez que, para a população de maior poder aquisitivo, as mudanças locais nos ecossistemas podem não causar uma mudança significativa no seu acesso aos bens, pois podem ser comprados e/ou importados, algumas vezes a preços artificialmente baixos. Já para comunidades locais com baixo perfil socioeconômico e muitas vezes sem subsídios governamentais, essas alterações podem representar um sério comprometimento do seu bem-estar (MEA, 2005).

Quanto maior o entendimento do que se tem a perder quando se substitui uma área natural por um sistema artificial (o chamado trade-off), melhor deverá ser a qualidade da decisão a ser tomada. Além disso, nem sempre a simples monetarização do que se está "perdendo" ou do que se vai "ganhar" basta para uma tomada de decisão bem fundamentada, já que às vezes uma pergunta mais importante a se fazer envolve "quem ganha" e "quem perde". Ainda, quando observada a insuficiência de determinado SE, torna-se possível focar a gestão na recuperação dos ecossistemas a eles associados, assim como no controle dos drivers de pressão sobre eles incidentes.

É importante ressaltar a importância de um mapeamento que represente a abrangência dos ecossistemas, assim como sua interação com a pressão antrópica, as demandas e os usos, dando condição à espacialização dos SEs. Os limites das áreas provedoras de SEs não necessariamente coincidem com os limites dos ecossistemas, inclusive são notáveis as sobreposições entre os mesmos SEs em diferentes ecossistemas. Mesmo essas áreas não traduzem a complexidade do mapeamento dos SEs, já que esse mapeamento deve incluir, além das áreas provedoras, ao menos as demandas, os usos e os fluxos desses serviços (Erhard et al., 2017). Assim, torna-se necessário, em uma próxima etapa, o mapeamento dos SEs da Ilha de Santa Catarina, além do mapeamento aqui apresentado. Este mapeamento dos SEs será a principal ferramenta tanto para se mensurar o atual capital natural em um momento de decisão como na tradução dessa informação para o público em geral.

Quanto maiores forem a disponibilidade e o entendimento dessas informações pela sociedade, melhor será a governança na gestão. Assim, o aporte de informações apresentado em uma linguagem acessível às mais diversas pessoas, além de dar subsídio para a implantação de uma GBE, trilha um caminho para uma maior governança.

Desse modo, conclui-se que a presente pesquisa representa uma etapa fundamental para espacialização dos SEs da Ilha de Santa Catarina e entorno. Além disso, conhecer os SEs básicos para o bem-estar humano pode garantir um direcionamento inicial para a implantação da gestão costeira integrada com base em ecossistemas, pois conhecer 
a importância e a complexidade dos ecossistemas é o principal passo para a busca de um desenvolvimento sustentável.

\section{Agradecimentos}

Aos membros do Laboratório de Gestão Costeira Integrada (LAGECI) da Universidade Federal de Santa Catarina (UFSC), Florianópolis. À Coordenação de Aperfeiçoamento de Pessoal de Nível Superior (Capes) pela concessão de bolsa de doutorado.

\section{Referências}

Asmus, M. L.; Kitzmann, D. Gestão Costeira no Brasil: estado atual e perspectivas. Montevidéu: Ecoplata, 2004.

Asmus, M. L.; Kitzmann, D.; Laydner, C.; Tagliani, C. R. A. Gestão Costeira no Brasil: instrumentos, fragilidades e potencialidades. Gestão Costeira Integrada, 5(4), 52-58, 2006.

Ban, S .S.; Pressey, R. L.; Graham, N. A. J. Assessing the effectiveness of local management of coral reefs using expert opinion and spatial Bayesian modeling. PLoS One, 10, 1-16, 2015. doi: 10.1371/journal.pone.0135465

Barragán, J. M. Política, Gestión y Litoral: una nueva visión de la gestión integrada de áreas litorales. Madrid, ES: Editorial Tébar Flores, 2014.

Böhnke-Henrichs, A.; Baulcomb, C.; Koss, R.; Hussain, S. S., de Groot, R. S. Typology and indicators of ecosystem services for marine spatial planning and management. Journal of Environmental Management, 130, 135-145, 2013. doi: 10.1016/j.jenvman.2013.08.027

Braat, L. C.; de Groot, R. S. The ecosystem services agenda: bridging the worlds of natural science and economics, conservation and development, and public and private policy. Ecosystem Services Journal, 1, 4-15, 2012. doi: 10.1016/j. ecoser.2012.07.011

Brasil. Lei.$^{\circ}$ 7.661, de 16 de maio de 1988. Institui o Plano Nacional de Gerenciamento Costeiro e dá outras providências: DOU de 18/5/1988.

Brasil. Decreto $n .^{\circ} 5.300$, de 7 de dezembro de 2004. Regulamenta a Lei n. ${ }^{\circ}$ 7.661, de 16 de maio de 1988, que institui o Plano Nacional de Gerenciamento Costeiro - PNGC, dispõe sobre regras de uso e ocupação da zona costeira e estabelece critérios de gestão da orla marítima, e dá outras providências. Brasília: DOU de 8/12/2004.

Christofoletti, A. Modelagem de Sistemas Ambientais. São Paulo: Edgard Blücher, 1999.

Cicin-Sain, B.; Knecht, R. W. Integrated coastal and ocean management: Concepts and Practices. Washington, D.C.: Island Press, 1998.

CIRM - Comissão Interministerial para os Recursos do Mar. Resolução n. ${ }^{\circ} 005$, de 03 de dezembro de 1997. Brasília, 1997.

Colombo, A. F.; Joly, C. A. Brazilian Atlantic Forest lato sensu: the most ancient Brazilian forest, and a biodiversity hotspot, is highly threatened by climate change. Revista Brasileira de Biologia, 70, 697-708, 2010. doi: 10.1590/ S1519-69842010000400002

Costanza, R.; D’Arge, R.; de Groot, R.; Farber, S.; Grasso, M.; Hannon, B.; Limburg, K.; Naeem, S.; O’Neill, R. V.; Paruelo, J.; Raskin, R. G.; Sutton, P.; van den Belt, M. The value of the world's ecosystem services and natural capital. Nature, 387, 253-260, 1997. doi: 10.1038/387253a0

De Groot, R. S.; Alkemade, R.; Braat, L.; Hein, L.; Willemen, L. Challenges in integrating the concept of ecosystem services and values in landscape planning, management and decision making. Ecological Complexity, 7, 260-272, 2010. doi: 10.1016/j.ecocom.2009.10.006

De Groot, R. S.; Wilson, M. A.; Boumans, R. M. J. A typology for the classification, description and valuation of ecosystem functions, goods and services. Ecological Economics, 41, 393-408, 2002. doi: 10.1016/S0921-8009(02)00089-7

Diederichsen, S. D.; Gemael, M. K.; Hernandez, A. de O.; Oliveira, A. O.; Paquette, M. L.; Schmidt, A. D.; Silva, P. G.; Silva, M. S.; Scherer, M. E. G. Gestão costeira no 
município de Florianópolis, SC, Brasil: um diagnóstico. Revista de Gestão Costeira Integrada, 13, 499-512, 2013. doi: $10.5894 / \mathrm{rgci} 425$

Erhard, M.; Banko, G.; Malak, D. A.; Martin, F. S. Mapping ecosystem types and conditions, In: Burkhard, B.; Maes, J. (Eds.). Mapping Ecosystem Services. Sófia: Pensoft Publishers, 2017. p. 77-82.

Ferretti, O. E.; Beltrame, A. V. Análise da gestão e manejo das unidades de conservação (UC) e de outras áreas protegidas e a criação da reserva da biosfera em ambiente urbano (RBAU) na Ilha de Santa Catarina. In: Anais do XVI Encontro Nacional dos Geógrafos. Porto Alegre, 2010. p. 1-12.

Figueiroa, A. C.; Brasil, G.; Pellin, A.; Scherer, M. E. G. Avaliação da efetividade da integração das Unidades de Conservação federais marinho-costeiras de Santa Catarina. Desenvolvolvimento e Meio Ambiente, 38, 361-375, 2016. doi: 10.5380/dma.v38i0.46974

Figueiroa, A. C.; Scherer, M. E. G. Para onde estamos indo? Uma avaliação do plano diretor do Município de Florianópolis para o entorno da Estação Ecológica de Carijós. Desenvolvolvimento e Meio Ambiente, 38, 283-301, 2016. doi: 10.5380/dma.v38i0.47110

Granek, E. F.; Polasky, S.; Kappel, C. V.; Reed, D. J.; Stoms, D. M.; Koch, E. W.; Kennedy, C. J.; Cramer, L. A.; Hacker, S. D.; Barbier, E. B.; Aswani, S.; Ruckelshaus, M.; Perillo, G. M. E.; Silliman, B. R.; Muthiga, N.; Bael, D.; Wolanski, E. Ecosystem services as a common language for coastal ecosystem-based management. Conservation Biology, 24, 207-216, 2010. doi: 10.1111/j.1523-1739.2009.01355.x

Gruber, N. L. S.; Strohaecker, T.; Ayup-Zouain, R. N.; Farina, F. Subsidios à Gestão Costeira: vulnerabilidades ambientais e aspectos legais para normativas de uso e ocupação. In: López, R. A. (Ed.). Problemática de Los Ambientes Costeros; Sur de Brasil, Uruguay y Argentina. Editorial Croquis, 2011. p. 41-55.

Hein, L.; Van Koppen, K.; de Groot, R. S.; Van Ierland, E. C. Spatial scales, stakeholders and the valuation of ecosystem services. Ecological Economics, 57, 209-228, 2006. doi: 10.1016/j.ecolecon.2005.04.005

ICMBio - Instituto Chico Mendes de Conservação da Biodiversidade. Dados Vetoriais Limites das Unidades de
Conservação Brasileiras. Brasília, 2016. Disponível em: $<$ http://www.icmbio.gov.br/portal/geoprocessamentos/ 51-menu-servicos/4004-downloads-mapa-tematico-e-dados-geoestatisticos-das-uc-s>. Acesso em: maio 2017.

Klein, A. H. F.; Short, A. D.; Bonetti, J. Santa Catarina Beach Systems, In: Short, A. D.; Klein, A. H. F. (Eds.). Brazilian Beach Systems. Springer International Publishing, 465-506, 2016. doi: 10.1007/978-3-319-30394-9

Krueger, T.; Page, T.; Hubacek, K.; Smith, L.; Hiscock, $\mathrm{K}$. The role of expert opinion in environmental modelling. Environmental Modelling \& Software, 36, 4-18, 2012. doi: 10.1016/j.envsoft.2012.01.011

McLeod K.L.; Lubchenco J.; Palumbi S.R.; Rosenberg A.A.. 2005. Communication Partnership for Science and the Sea scientific consensus statement on marine ecosystem-based management. www.compassonline.org/pdf files/EBM Consensus_Statement_v12.pdf. Viewed 23 May 2007.

Martin, T. G.; Burgman, M. A.; Fidler, F.; Kuhnert, P. M.; Low-Choy, S.; Mcbride, M.; Mengersen, K. Eliciting Expert Knowledge in Conservation Science. Conservation Biology, 26, 29-38, 2012. doi: 10.1111/j.1523-1739.2011.01806.x

MEA - Millennium Ecosystem Assessment. Ecosystems and human well-being: Synthesis. Washington, DC: Island Press, 2005. doi: 10.1196/annals. 1439.003

MMA - Ministério do Meio Ambiente. Projeto Terra Mar, 2015. Brasília. Disponível em: <www.mma.gov.br/gestao-territorial/projeto-terramar>. Acesso em: maio 2017.

Neal, W. J.; Pilkey, O. H.; Cooper, A. G.; Longo, N. J. Why coastal regulations fail. Ocean and Coastal Management, no prelo, 2017. doi: 10.1016/ j.ocecoaman.2017.05.003

Odum, E. Fundamentals of Ecology. Philadelphia: Sounders, 1956.

Oliveira, K. A.; Corona, H. M. P. A percepção ambiental como ferramenta de propostas educativas e de políticas ambientais. Anap Brasil, 1, 53-72, 2008.

Oliveira, T. C. R.; Scherer, M. E. G.; Anfuso, G.; Almeida, F .B.; Diederichsen, S. D.; Williams, A. Classificação dos cenários costeiros de praias da Ilha de Santa Catarina, Florianópolis - Brasil. Desenvolvimento e Meio Ambiente, 39, 217-229, 2016. doi: 10.5380/dma.v39i0.46171 
Scarano, F. R.; Ceotto, P. Brazilian Atlantic forest: impact, vulnerability, and adaptation to climate change. Biodiversity and Conservation, 24, 2319-2331, 2015. doi: 10.1007/ s10531-015-0972-y

Scherer, M. E. G.; Sanches, M.; Negreiros, D. H. Gestão das zonas costeiras e as políticas públicas no Brasil: um diagnóstico. In: Barragán Muñoz, J. M. (Ed.). Manejo Costero Marino Integrado y Política Pública en Iberoamérica: un diagnostico. Necesidad de cambio. Cádiz: Red Ibermar, 2010. p. 291-330.

Scherer, M. E. G.; Asmus, M. L. Ecosystem-Based Knowledge and Management as a tool for Integrated Coastal and Ocean Management: A Brazilian Initiative. Journal of Coastal Research, 690-694, 2016. doi: 10.2112/SI75-138.1

SDS - Secretaria de Desenvolvimento Sustentável. Sistema de Informações Geográficas de Santa Catarina (SIGSC). Florianópolis, 2010. Disponível em $<$ http://sigsc.sds.sc.gov. br/>. Acesso em: maio 2017.
Tomazzoli, E. R.; Pellerin, J. M. Unidades do mapa geológico da ilha de Santa Catarina: as rochas. Geosul, 30, 225-248, 2015.

Traun, C.; Klug, H.; Burkhard, B. Mapping techniques. In: Burkhard, B.; Maes, J. (Eds.). Mapping Ecosystem Services. Sófia: Pensoft Publishers, 2017. p. 57-64.

UN - United Nations. World Urbanization Prospects: The 2011 Revision. New York, 2010. doi: 10.2307/2808041

Veiga Lima, F. A.; Almeida, F. B.; Torres, R. P.; Scherer, M. E. G. Modelo conceitual de avaliação de ameaças sobre serviços ecossistêmicos de sistemas de dunas. Estudo de caso: os campos de dunas da Ilha de Santa Catarina/SC , Brasil. Desenvolvolvimento e Meio Ambiente, 38, 199-211, 2016. doi: 10.5380/dma.v38i0.46992 